\title{
ARQUITETURA DA INFORMAÇÃO ANALÍTICA PARA INTEGRAÇÃO DE DADOS DA PESQUISA E PÓS-GRADUAÇÃO: UM ESTUDO DE CASO DA UNIVERSIDADE DO ESTADO DA BAHIA
}

\author{
ANALYTICAL INFORMATION ARCHITECTURE FOR THE \\ INTEGRATION OF RESEARCH AND GRADUATE \\ PROGRAMS DATA: A CASE STUDY FROM BAHIA \\ STATE UNIVERSITY
}

\author{
Eduardo Manuel de Freitas Jorge \\ Franciele Portugal dos Santos ${ }^{b}$ \\ Breno Pádua Brandão Carneiroc
}

Fernanda Almeida Machadod

\begin{abstract}
RESUMO
Introdução: As universidades precisam monitorar os seus indicadores e informações para elaboração de estratégias de estímulo à pesquisa e pós-graduação. A Universidade do Estado da Bahia (UNEB) tem um ambiente similar a outras universidades onde existe uma dispersão dos dados devido à falta de integração de sistemas internos e externos, tais como os sistemas da Plataforma Lattes e da Plataforma Sucupira do Conselho Nacional do Desenvolvimento Científico e Tecnológico (CNPq) e da Coordenação de Aperfeiçoamento de Pessoal de Nível Superior (CAPES) respectivamente. A redundância de dados, informações inconsistentes e não consolidadas dificultam os gestores no processo de correlacionamento de dados para análise dos principais indicadores de avaliação. Objetivo: Uma proposta de arquitetura da informação materializada em solução analítica para integração e visualização de dados relevantes para o mapeamento do cenário da Pesquisa e Pós-Graduação da UNEB. Metodologia: Para este projeto a metodologia está fundamentada na pesquisa experimental e na metodologia de estudo de caso. Estratégias como a matriz de necessidade para o levantamento da camada de informação e a etapa de retroalimentação através de entrevistas com os gestores da

\footnotetext{
a Doutor em Difusão do Conhecimento no programa multi institucional pela Universidade Federal da Bahia - UFBA. Email: emjorge1974@gmail.com

b Formanda do curso de Sistemas de Informação na UNEB (Universidade do Estado da Bahia). Email: franciele.portugal@gmail.com

c Doutor em Educação e Contemporaneidade pela Universidade do Estado da Bahia - UNEB. Email: bcarneiro@uneb.br

d Mestra em Arquitetura, Tecnologia e Cidade pela UNICAMP e Especialista em Gerenciamento BIM pelo SENAI CIMATEC, com Bacharelado em Arquitetura e Urbanismo pela UFBA. Email: famachado@uneb.br
} 
UNEB se somaram a etapas de elaboração do plano provisório do assunto, bem como a busca por fontes de dados e criação e desenvolvimento do Sistema de Apoio à Decisão (SAD). Resultados: Obteve-se um modelo de dados com três cubos temáticos materializados em painéis com metáforas visuais em tabelas e gráficos interativos permitindo filtros e consultas em drill. Conclusões: A materialização de uma solução de gestão analítica se mostrou relevante nas aferições dos gestores que buscam potencializar os indicadores de pesquisa da UNEB. Com o SAD desenvolvido, os gestores da UNEB podem agora fazer campanhas mais precisas de estímulo à pesquisa em regiões ou departamentos.

Descritores: Arquitetura da informação. Sistema de Apoio à Decisão. Indicadores Acadêmicos.

\section{INTRODUÇÃO}

O sistema brasileiro de pós-graduação Stricto Sensu evoluiu de forma significativa nos últimos anos, quase dobrando o número total de cursos entre 1998 e 2011, considerando as Instituições de Ensino Superior (IES) públicas e privadas. Nesse período, a taxa de crescimento de alunos matriculados em cursos de pós-graduação se apresentou expressiva. Em 2010, por exemplo, verifica-se um incremento de $128 \%$ em relação às matrículas de 1998 , sendo que o percentual de matrículas nas IES públicas é superior ao das IES privadas (CIRANI; CAMPANARIO; SILVA, 2015).

No que tange ao planejamento do sistema de Pós-graduação, o Plano Nacional de Pós-Graduação (PNPG) 2011-2020 (CAPES, 2018) destaca a inovação como um novo paradigma e fator decisivo para o desenvolvimento. De acordo com o referido Plano, "Um ambiente que favoreça a inovação nas empresas, no país, é induzido pela existência de ciência avançada e pela capacidade regional de formar recursos humanos de ponta, mesmo que estas últimas atividades tenham seus centros de atividades na academia" (CAPES, 2018; p. 181).

A perspectiva do PNPG 2011-2020, portanto, atribui à academia, por meio de seus programas de pós-graduação, papel importante na produção de conhecimento e formação de recursos humanos de ponta, capazes de promover a inovação nas empresas e no mercado, de modo mais abrangente. Assim, a avaliação da produção de conhecimento e inovação na pós-graduação se 
reveste de renovada importância no que tange aos horizontes almejados pelo sistema nacional de pós-graduação.

No que concerne a avaliação da pós-graduação, de modo particular, a Proposta de Aprimoramento do Modelo de Avaliação da PG, Documento Final da Comissão Nacional de Acompanhamento do PNPG 2011-2020 (CAPES, 2018) chama atenção para o fato da inovação brasileira não ter acompanhado o desenvolvimento da produção científica. O documento aponta a 64a posição ocupada pelo Brasil no Índice Global de Inovação em 2018 e a relativa inércia no crescimento de patentes depositadas por residentes do Brasil no país e alhures como indícios do descompasso.

Frente a esse cenário, o documento supracitado aponta dois focos principais de atuação da Pós-graduação:

O avanço do conhecimento, incluindo a formação de docentes e pesquisadores para as Universidades e para o meio científicoacadêmico, e a atuação e impacto no desenvolvimento econômico e social (incluindo a interação com segmento empresarial e a atuação na área pública e na formulação de políticas públicas) (CAPES, 2018; p. 8, grifo nosso)

Dentre os temas contemplados na Proposta de Aprimoramento do Modelo de Avaliação da PG (CAPES, 2018), sistematizados a partir de ampla consulta a entidades científicas e acadêmicas ${ }^{1}$, figuram a auto avaliação e a inovação como proposições novas. A perspectiva da proposta aponta que a inovação deve ser abordada a partir de "indicadores que avaliem a capacidade de apoio que os Programas de Pós-Graduação possam oferecer às empresas em seus processos de inovação" (CAPES, 2018).

Embora o Documento Final da Comissão Nacional de Acompanhamento do PNPG 2011-2020, aponte a valorização da auto avaliação como perspectiva para a avaliação dos programas de pós-graduação, o documento indica que não há orientações fornecidas externamente, pela CAPES ou qualquer outra entidade especializada no assunto.

Frente a esse cenário, verifica-se que, não obstante as IES serem

\footnotetext{
1 ABC, ANDIFES, ABRUEM, ABRUC, CNE, CONFAP, CONSECTI, CNPq, CTC-ES, FINEP, INEP, FOPROP, MCTIC, MDIC e SBPC.
} 
instadas pela CAPES a desenvolver processos de auto avaliação e utilizar indicadores da produção de conhecimento e inovação, faltam referências e orientações oficiais. Dentre as referências alternativas às orientações de órgãos oficiais, as metodologias utilizadas por institutos que desenvolvem rankings das instituições de ensino superior se apresentam como opção.

Um exemplo externo a CAPES de avaliação é o Ranking Universitário Folha RUF (2017) que avalia as IES brasileiras com base em indicadores relacionados a aspectos das áreas de pesquisa, ensino, mercado, internacionalização e inovação com o objetivo de mensurar quantitativamente a qualidade das IES. Há, também, índices globais, como o Centro de Rankings Universitários Mundiais (CWUR) (CWUR, 2018) que possui uma metodologia similar avaliando indicadores relacionados à pesquisa, ensino, mercado e internacionalização.

Ambos os índices atribuem importância à produção científica. No índice RUF a pesquisa compreende $45 \%$ da nota global, enquanto no índice CWUR a pesquisa compreende $55 \%$ do valor da nota global. De acordo com dados colhidos para avaliar o indicador de qualidade inovação, presente no índice RUF (2017), apenas $68,20 \%$ das 195 IES públicas e privadas classificadas para o ranking em 2017 solicitaram patentes na última década.

Observa-se que a Universidade do Estado da Bahia (UNEB) tem um ambiente similar a outras universidades quanto a falta de informações para potencializar os indicadores em relação à pesquisa. O autor Barbosa (2018) já discutia essa problemática, dando ênfase ao ensino na graduação, apresentando uma estudo acerca do desenvolvimento de uma ferramenta tecnológica de apoio à gestão acadêmica na UNEB que sugere a dispersão dos dados da universidade que ocorre não só devido à falta de integração entre os diversos sistemas internos utilizados, mas também pela falta de integração com os sistemas externos, tais como: Plataforma Sucupira da CAPES, Diretório de Grupos de Pesquisa (DGP) e Plataforma Lattes do Conselho Nacional de Desenvolvimento Científico e Tecnológico (CNPq). Essa situação, verificada pelo autor e que persiste até o momento, sugere um cenário de sistemas com 
redundância de dados, informações inconsistentes e não consolidadas, dificultado assim a realização, pelos gestores da UNEB, de consultas analíticas de dados correlacionados com os principais indicadores de avaliação.

No que tange às organizações, de modo geral, o impacto negativo da falta de dados para a tomada de decisão já era um tema relevante desde os anos 70 , quando surgiu a necessidade de criar e desenvolver sistemas que centralizam informações para o nível tático e estratégico das organizações. Nesse contexto, o termo Business Intelligence $(\mathrm{BI})$ emergiu conectado aos Sistemas de Apoio à Decisão (SADs), incorporando metodologias analíticas e modelos para extrair informações relevantes para as organizações. Os SADs visam prover soluções através da integração de dados internos e externos à organização, que sejam proveitosas para seu planejamento estratégico e processo de tomada decisão (VERCELLIS, 2009).

Neste contexto, este artigo apresenta uma proposta de arquitetura da informação materializada em solução analítica para integração e visualização de dados relevantes para o mapeamento do cenário da Pesquisa, Inovação e PósGraduação da UNEB. Para a concepção da solução analítica foram realizadas etapas de levantamento de requisitos e fontes de dados, definição de indicadores, modelagem de dimensões e suas hierarquias, cubos de dados e 0 desenvolvimento de painéis de bordo. Essas etapas foram necessárias para o delineamento da arquitetura da informação da solução proposta e estão detalhadas nas seções seguintes.

\section{CENÁRIO DA UNEB NOS CAMPOS DA PESQUISA, INOVAÇÃO E PÓS- GRADUAÇÃO}

A UNEB é uma universidade multicampi com 29 departamentos localizados em 24 municípios baianos. Devido a sua capilaridade, a UNEB tem uma produção dispersa e volumosa de dados acadêmicos. A UNEB iniciou suas atividades de pós-graduação stricto sensu em 2001 com o Programa Educação e Contemporaneidade (PPGEduC/UNEB), ofertando curso de mestrado. Em dezoito anos, a Instituição expandiu essa oferta para 20 cursos em dezenove 
diferentes programas sediados em seis municípios do estado (Santo Antônio de Jesus, 2 cursos; Alagoinhas, 2 cursos; Jacobina, 1 curso; Juazeiro, 2 cursos; Paulo Afonso, 2 cursos; Salvador, 11 cursos). Hoje são ofertados cursos de mestrado (profissional e acadêmico) e doutorado nas áreas de educação, ciências agrárias, química, letras/linguística, história, biodiversidade, ensino, farmácia e interdisciplinar. A figura 1, permite visualizar como a expansão da oferta de cursos de pós-graduação stricto sensu ocorreu no período de 2001 a 2018.

Figura 1 - Evolução de cursos de Pós-Graduação Stricto Sensu UNEB.

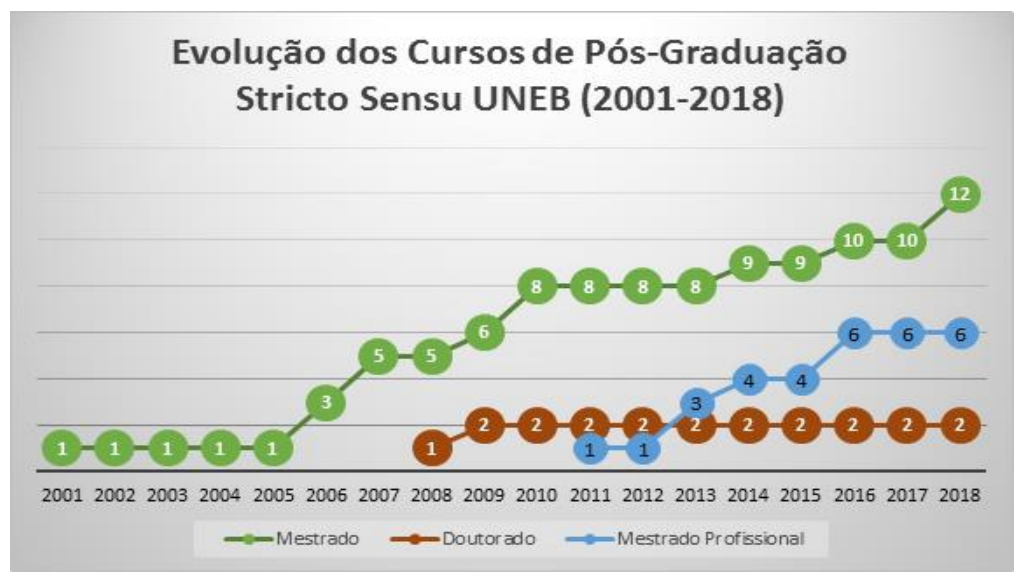

Fonte: Elaborada pelos autores a partir de dados da Plataforma Sucupira (CAPES, 2017).

Em 2006, cinco anos após a implantação do primeiro programa de mestrado na área de educação, foram implantados dois programas nas áreas de química e letras/linguística. Nos anos seguintes, a expansão se manteve de forma regular, com novos cursos de mestrado nas áreas de história, ciências agrárias, interdisciplinar, letras/linguística, educação, biodiversidade, ensino, astronomia/física e farmácia, bem como, a implantação de dois cursos de doutorado.

A partir de 2011, a UNEB passa a ofertar, também, cursos de mestrado profissional. Atualmente, são ofertados seis cursos de mestrado profissional, nas áreas de educação, letras/linguística, história e astronomia/física que atendem, substancialmente, profissionais da rede de educação básica. Esse conjunto de programas de pós-graduação é responsável por uma expressiva produção 
intelectual qualificada. Além da atuação em programas de pós-graduação, outras ações dos pesquisadores da UNEB são a participação em grupos de pesquisa, produções de artigos qualificados e patentes e a realização de projetos de pesquisa.

\section{TRABALHOS CORRELATOS}

Esta seção apresenta trabalhos e ferramentas relacionadas a soluções de $\mathrm{BI}$ na área acadêmica. As primeiras pesquisas analisadas foram as ferramentas criadas por Oliveira (2013) e Barbosa (2018) para auxiliar na gestão da UNEB, as mesmas seguiram áreas acadêmicas distintas. $O$ pesquisador Oliveira (2013) estudou a área voltada para aprendizagem dos discentes de graduação de EAD (Ensino a Distância), que por sua vez utilizam - Ambiente Virtual de Aprendizagem (AVA) da universidade na ferramenta MOODLE (Modular Object-Oriented Dynamic Learning Environment). Já Barbosa (2018) estudou as dificuldades do processo de tomada de decisão no contexto da avaliação das IES na UNEB, assim propôs uma ferramenta para auxiliar na disseminação de informações aos gestores para apoiar os processos de avaliação institucional.

Ambos os trabalhos estudaram indicadores com 0 objetivo de fundamentar as suas ferramentas. O pesquisador Barbosa (2018) estudou indicadores voltados para a qualidade da educação superior enquanto que Oliveira (2013) estudou indicadores que fossem capazes de auxiliar o docente ou tutor no acompanhamento e avaliação dos discentes com base nas interações realizadas no AVA. A maior diferença entre ambos nessa etapa é que Oliveira (2013) utilizou a técnica de criação própria chamada matriz de necessidades para mapear os dados que seriam necessários para construção do Data Warehouse (DW), como também foi realizado nesta pesquisa.

A metodologia adotada por Oliveira (2013) também apresentou semelhanças a proposta desta pesquisa durante seu processo de desenvolvimento. Basicamente, Oliveira (2013) desenvolveu o seu trabalho em 6 fases. A primeira fase se trata do levantamento de requisitos com o estudo dos 
indicadores e a definição das visões para realizações de consultas e visualização de relatórios e gráficos com base nos indicadores identificados. A segunda fase se trata do projeto lógico do banco de dados, ou seja, a modelagem multidimensional do DW. A terceira fase se trata do projeto físico do banco de dados e construção/customização da ferramenta proposta, ou seja, a consolidação do DW e construção da ferramenta OLAP (Online Analytical Processing) com as visões gerenciais para visualização dos dados. Por fim, as duas próximas fases consistiram na homologação dos dados carregados para validação da solução proposta e na definição do ambiente de produção, onde os ajustes só seriam realizados na sexta e última fase de manutenção e atualização da ferramenta.

A ferramenta Experta (2018) e o trabalho de Vallejera, Lozano-Díaz, Rodriguez-Sánchez (2016) também apresentam características muito próximas do trabalho desenvolvido nesta pesquisa por focar principalmente nas áreas de pesquisa e inovação, que são áreas incorporadas neste trabalho. $O$ trabalho de Vallejera, Lozano-Díaz, Rodriguez-Sánchez (2016) foi construir uma ferramenta de BI para dar suporte a tomada de decisão na unidade acadêmica de ciência e tecnologia da Universidade de Cuba. Foi utilizada a metodologia bibliométrica que é uma área instrumental para avaliação da atividade científica usada para determinar o comportamento de determinados aspectos que são considerados pelos programas acadêmicos para financiar projetos de pesquisa e inovação, assim como políticas institucionais para conceder melhores vagas nos programas de pós-graduação, permitindo validar os programas de doutorados. A ferramenta Experta (2018) é uma plataforma desenvolvida com o objetivo de gerar relatórios e indicadores dinamicamente quanto a capacidade e qualidade da produção técnico-científica da instituição. Além disso, a plataforma também permite estruturar novos cursos na instituição, pois facilita a identificação de especialistas com a expertise necessária para dar suporte nesse processo, assim como grupos de pesquisa, produções e projetos. A solução proposta se assemelha a ferramenta Experta pela carga e integração de dados dos pesquisadores da plataforma Lattes e Sucupira, e se diferencia pois teve seu 
enfoque nos indicadores segmentados por região e departamento, visto que a UNEB é uma universidade multicampi.

Outro trabalho que engloba funcionalidades da ferramenta Experta (2018) e utiliza também a metodologia bibliométrica como Vallejera, LozanoDíaz, Rodriguez-Sánchez (2016) foi realizado por Magalhães et al. (2014), o qual propõe um modelo para mineração de dados para mapeamento de competências essenciais em uma determinada área da ciência correlacionando-as com a expertise de pesquisadores. Para validar o modelo realizou uma análise da produção científica através das bases Lattes e DGP do CNPq para identificar especialistas que estudam a doença Dengue, extraindo dados relacionados a geolocalização, publicações, orientações, entre outros aspectos.

\section{METODOLOGIA}

O arcabouço metodológico para este projeto está fundamentado na pesquisa experimental e baseada na metodologia de estudo de caso (GIL, 2017). Na presente pesquisa, busca-se um percurso metodológico para modelar uma camada de informação como fonte integrada de dados para uma solução analítica que permita a visualização de dados relevantes do cenário da Pesquisa, Inovação e Pós-Graduação da UNEB. O percurso preconiza 5 (cinco) etapas:

- (i) Elaboração do Plano Provisório do Assunto: nesta etapa foi realizada a pesquisa documental para formulação dos principais indicadores de avaliação das universidades que regem a Pesquisa, Inovação e Pós-Graduação.

- (ii) Busca das Fontes: nesta etapa foram definidas as principais bases de dados externas, são elas: Diretório de Grupos de Pesquisa do CNPQ, Plataforma Sucupira da CAPES, plataforma do Instituto Nacional da Propriedade Industrial e Plataforma Lattes. Outro ponto foi a busca por bases de dados dos sistemas internos da UNEB. As bases pesquisadas foram: o Sistema Online de Iniciação Científica e 
o Sistema Integrado de Planejamento responsável pelo registro e acompanhamento dos projetos de pesquisa da UNEB. Foram obtidas, também, informações da estrutura departamental da UNEB por região e cidade e informações dos seus pesquisadores através de planilhas em Excel fornecidas pelos setores Secretaria Especial de Avaliação Institucional e Pró-Reitoria de Gestão e Desenvolvimento de Pessoas.

- (iii) Elaboração de Matriz de Necessidades: para modelar a necessidade de informações analíticas e como estas foram mapeadas para esquemas de dados, utilizou-se a estratégia de aplicação da matriz de necessidades proposta por Oliveira (2013). Para construir a matriz de necessidades foi realizada a análise das fontes de dados e foram realizadas, também, entrevistas, parcialmente estruturadas, com profissionais do setor da Pró-Reitoria de Pesquisa e Ensino de Pós-Graduação (PPG);

- (iv) Criação e Desenvolvimento do Artefato: nesta etapa foi definida a ferramenta OLAP para o mapeamento e visualização dos dados. Dentre algumas opções, definiu-se pela ferramenta de código aberto Knowage (KNOWAGE, 2018), utilizando o banco de dados MariaDB (MARIADB, 2018). Além disso, nesta etapa foi modelado o DW com suas dimensões, hierarquias e fatos. Com o DW modelado foi implementado o processo de ETL (Extração, Transformação e Carga) integrando as fontes de dados identificadas na etapa II no mesmo. Umas das principais fontes foram os dados da Plataforma Lattes onde foram importados os principais dados dos 2.353 docentes da UNEB. A ferramenta Knowage foi utilizada para criar uma série de painéis de bordo temáticos associados com os indicadores identificados na etapa I e baseados nas necessidades dos gestores da UNEB observadas na etapa III.

- (v) Avaliação do Artefato: nesta etapa foi realizada a análise de resultados e a retroalimentação dos painéis de bordo modelados na 
etapa iv. Para isso, a ferramenta analítica proposta foi apresentada para os seguintes gestores: Pró-Reitora de Pesquisa e Ensino de Pós-Graduação, Gerente de Pesquisa, Gerente de Pós-Graduação, Coordenador da Agência UNEB de Inovação e a SEAVI (Secretaria Especial de Avaliação Institucional). Nesta dinâmica foi obtido o feedback dos gestores com objetivo da geração de uma nova versão da ferramenta analítica contemplando as observações dos gestores UNEB.

\section{INDICADORES PARA AVALIAÇÃO DA PESQUISA, INOVAÇÃO E PÓS- GRADUAÇÃO}

Para compreender como as áreas da pesquisa, inovação e pósgraduação são avaliadas foi realizada uma pesquisa sobre os principais indicadores de avaliação de desempenho das universidades. Os indicadores irão nortear quais informações devem ser monitoradas pelos gestores acadêmicos da UNEB. Essa pesquisa foi organizada em quadros, onde 0 Quadro 1 é referente à pesquisa e o Quadro 2 à pós-graduação. Nos Quadros 1 e 2, a coluna "Incorporado" corresponde os indicadores priorizados no escopo desta pesquisa.

O quadro 1 demonstra como as universidades são avaliadas em relação a pesquisa. Aspectos como a produção científica do docente é um dos indicadores fundamentais para acompanhar a produção da pesquisa nas universidades. Outro indicador são as citações de artigos, também conhecido como Índice $\mathrm{H}$, para o índice RUF este é um indicador relevante. Outros indicadores observados na pesquisa são recursos recebidos, produções de teses e bolsistas de produtividade. Neste primeiro ciclo da arquitetura da informação para solução de $\mathrm{Bl}$, posto neste artigo, foi incorporado somente os indicadores publicações por docente e bolsista de produtividade. 
Eduardo Manuel de Freitas Jorge, Franciele Portugal dos Santos, Breno Pádua Brandão Carneiro, Fernanda Almeida Machado

Arquitetura da Informação Analítica para Integração de Dados da Pesquisa e Pós-Graduação: Um Estudo de Caso da Universidade do Estado da Bahia

Quadro 1 - Indicadores de Pesquisa

\begin{tabular}{|c|c|c|}
\hline \multicolumn{3}{|c|}{ Pesquisa } \\
\hline Indicador & Descrição & $\begin{array}{l}\text { Incorporad } \\
\quad 0\end{array}$ \\
\hline Total de publicações & $\begin{array}{l}\text { Total de publicações de artigos científicos pela } \\
\text { universidade em periódicos (Web of Science). }\end{array}$ & Não \\
\hline Total de citações & $\begin{array}{l}\text { Total de citações dos trabalhos científicos produzidos } \\
\text { pela universidade para mensurar a importância dos } \\
\text { mesmos. }\end{array}$ & Não \\
\hline Citações por publicação & $\begin{array}{l}\text { Quantidade média de citações por artigo científico } \\
\text { publicado pela universidade. }\end{array}$ & Não \\
\hline Publicações por docente & $\begin{array}{l}\text { Quantidade média de artigos científicos publicados por } \\
\text { docente. }\end{array}$ & Sim \\
\hline Citações por docente & $\begin{array}{l}\text { Quantidade média de citações que cada docente } \\
\text { recebeu. }\end{array}$ & Não \\
\hline $\begin{array}{l}\text { Publicações em revistas } \\
\text { nacionais }\end{array}$ & $\begin{array}{l}\text { Quantidade de artigos científicos publicados em revistas } \\
\text { nacionais pela universidade (SciELO). }\end{array}$ & Sim \\
\hline $\begin{array}{l}\text { Recursos recebidos por } \\
\text { instituição }\end{array}$ & $\begin{array}{l}\text { Valor médio de recursos financeiros obtidos através dos } \\
\text { órgãos de fomento por cada docente para medir à } \\
\text { assistência recebida para pesquisa. }\end{array}$ & Não \\
\hline Bolsistas CNPq & $\begin{array}{l}\text { Quantidade de docentes que são bolsistas de } \\
\text { produtividade do órgão de fomento CNPq. }\end{array}$ & $\operatorname{Sim}$ \\
\hline Teses & $\begin{array}{l}\text { Quantidade de teses defendidas pelo número de } \\
\text { docentes. }\end{array}$ & Não \\
\hline
\end{tabular}

Fonte: Adaptado de RUF (2017).

O Quadro 2 apresenta os indicadores propostos pela CAPES para avaliar os programas de Pós-Graduação. O indicador relativo ao Corpo Docente apresenta-se fundamental, já que impacta estruturalmente na universidade. Um curso de pós-graduação pode ser avaliado com notas de 1 à 7 , para formação da mesma são levados em consideração os aspectos do Quadro 2. Este tipo de avaliação é feita considerando os últimos quatro anos de produção do programa. Para compor a arquitetura da informação do Bl foram priorizadas nesta fase da pesquisa os indicadores relativos ao Corpo Docente e suas Produções Intelectuais. 
Eduardo Manuel de Freitas Jorge, Franciele Portugal dos Santos, Breno Pádua Brandão Carneiro, Fernanda Almeida Machado

Arquitetura da Informação Analítica para Integração de Dados da Pesquisa e Pós-Graduação: Um Estudo de Caso da Universidade do Estado da Bahia

\section{Quadro 2 - Indicadores de Pós-Graduação}

\begin{tabular}{|c|c|c|}
\hline \multicolumn{3}{|c|}{ Pós-Graduação } \\
\hline Indicador & Descrição & $\begin{array}{c}\text { Aspectos } \\
\text { Incorporados }\end{array}$ \\
\hline $\begin{array}{l}\text { Proposta do } \\
\text { Programa }\end{array}$ & $\begin{array}{l}\text { Avalia o quanto o programa está de acordo com a sua } \\
\text { proposta e o quanto a mesma está atualizada. Além de } \\
\text { verificar como o planejamento do programa alcançará seus } \\
\text { objetivos de forma a atender as necessidades internas e } \\
\text { externas e como o mesmo direciona a sua infraestrutura para } \\
\text { ensino, pesquisa e extensão. }\end{array}$ & Nenhum. \\
\hline Corpo Docente & $\begin{array}{l}\text { Avalia a distribuição dos docentes quanto à sua titulação, } \\
\text { formação e produção intelectual, que deve ser condizente à } \\
\text { proposta do programa. Além de avaliar a dedicação dos } \\
\text { docentes permanentes e a colaboração dos docentes no } \\
\text { ensino e pesquisa na graduação. }\end{array}$ & $\begin{array}{l}\text { Titulação, regime, } \\
\text { colaboração na } \\
\text { pesquisa e } \\
\text { obtenção de } \\
\text { financiamentos } \\
\text { pelos órgãos de } \\
\text { fomento. }\end{array}$ \\
\hline $\begin{array}{c}\text { Corpo Discente, } \\
\text { Teses e } \\
\text { Dissertações }\end{array}$ & $\begin{array}{l}\text { Verifica a quantidade de teses e dissertações defendida } \\
\text { quanto aos docentes permanentes e discentes, a disposição } \\
\text { das orientações quanto ao corpo docente e a eficiência do } \\
\text { programa na formação de mestres e doutores bolsistas. Além } \\
\text { de avaliar a qualidade das teses e dissertações e da produção } \\
\text { dos discentes na pós-graduação e graduação através de } \\
\text { publicações e indicadores relevantes para a área. }\end{array}$ & Nenhum. \\
\hline $\begin{array}{l}\text { Produção } \\
\text { Intelectual }\end{array}$ & $\begin{array}{l}\text { Avalia as produções intelectuais do programa e a distribuição } \\
\text { dos mesmos em relação ao corpo docente permanente. Além } \\
\text { disso, avalia também a produção técnica, patentes e outras } \\
\text { produções relevantes. }\end{array}$ & $\begin{array}{c}\text { Produções como } \\
\text { patentes, marcas } \\
\text { e softwares. }\end{array}$ \\
\hline Inserção Social & $\begin{array}{l}\text { Avalia o impacto regional e nacional do programa relacionado } \\
\text { à aspectos educacionais, sociais, científicos, tecnológicos, } \\
\text { entre outros. Além disso, avalia questões como parcerias } \\
\text { nacionais e internacionais consolidados pelos docentes, e a } \\
\text { apresentação do programa em meios digitais. }\end{array}$ & $\begin{array}{c}\text { Parcerias } \\
\text { internacionais. }\end{array}$ \\
\hline
\end{tabular}

Fonte: Adaptado de CAPES (2017)

\section{ARQUITETURA DA INFORMAÇÃO PARA SOLUÇÃO ANALÍTICA}

Como mencionado na introdução, o objetivo deste projeto é propor a arquitetura da informação necessária para estruturar um SAD com o foco nos gestores em atividades de planejamento estratégico e nas ações de estímulo à Pesquisa, Inovação e Pós-graduação da UNEB. A modelagem da camada de 
dados do SAD foi baseada nos indicadores pesquisados na seção anterior e na aplicação da técnica de Matriz de Necessidade proposta por (OLIVEIRA, 2013). Esta técnica é útil para mapear os dados de forma visual e interativa permitindo o delineamento da arquitetura da informação, onde as linhas representam descritores e as colunas representam métricas. Os dados da matriz foram coletados através de entrevistas, parcialmente estruturadas, com profissionais do setor da Pró-Reitoria de Pesquisa e Ensino de Pós-Graduação (PPG) da UNEB. Findada esta etapa, foi possível elaborar o quadro $3 \mathrm{com}$ os dados de interesse para o processo de tomada de decisão dos gestores acadêmicos.

Quadro 3 - Dados de Interesse Gestores UNEB.

\begin{tabular}{|l|l|l|}
\hline \multicolumn{1}{|c|}{ Área } & \multicolumn{1}{c|}{ Dados de interesse } \\
\hline Titulação x Regime de & - Quantidade de Docentes por Titulação \\
Trabalho & - Quantidade de Docentes por Regime de \\
& & Trabalho \\
& - Bolsistas Produtividade \\
\hline $\begin{array}{l}\text { Produtividade } \\
\text { Docente }\end{array}$ & - Número de Orientações de Iniciação \\
& - Científica \\
& - Qarticipação em Grupos de Pesquisa \\
& - Artigos Científicos Qualificados \\
& - Quantidade de Produções Intelectuais \\
\hline
\end{tabular}

Fonte: Elaborado pelos autores.

Além da titulação, regime de trabalho e produtividade docente foi identificado ainda que os gestores analisam os dados seguindo a hierarquia Região->Cidade$>$ Departamento->Docente. Esta navegabilidade é fundamental para compreender o cenário da UNEB que como apresentado na seção 2 é uma universidade multicampi. Outro elemento estruturante do modelo é a dimensão Ano que permite ao gestor fazer uma análise da evolução da universidade diante dos indicadores. Já o recorte temporal dos dados, a serem carregados, foi definido como de 5 (cinco) anos seguindo o direcionamento da CAPES (últimos 4 anos) e sendo acrescido de mais 1 (um) ano com base no planejamento estratégico da UNEB.

Como resultado foram modelados três cubos de dados: (i) Corpo Docente; (ii) Produções Qualificadas; (iii) Produtividade Docente. O primeiro cubo Corpo Docente está representado na figura 2 e utiliza as dimensões Região, Bolsistas Produtividade, Titulação e Regime. A partir dessas dimensões, a tabela fato Docentes pode produzir informações referentes aos indicadores do Corpo 
Docente, por exemplo, como os docentes estão distribuídos por Região$>$ Cidade->Departamento de acordo com o regime de trabalho e titulação ou como os docentes bolsistas de produtividade estão distribuídos e qual a situação dos mesmos em relação à titulação e ao regime dos outros docentes.

Figura 2 - Esquema com os elementos do cubo Corpo Docente

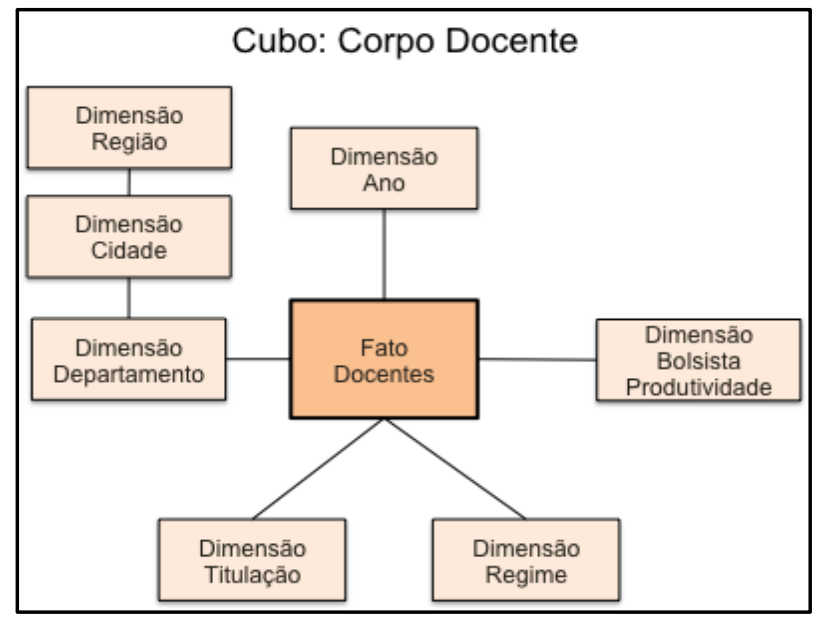

Fonte: Elaborada pelos autores

O segundo cubo Produções Qualificadas, apresentado na figura 3, é referente a quantidade de artigos publicados em periódicos dos docentes da UNEB nos últimos 5 (cinco) anos. Neste cubo todos os artigos são classificados segundo a área de conhecimento através da categorização qualis da CAPES. As co-autorias não são contabilizadas para um mesmo departamento visto que neste cubo a menor granularidade é o próprio departamento.

Figura 3 - Esquema com os elementos do cubo Produções Qualificadas.

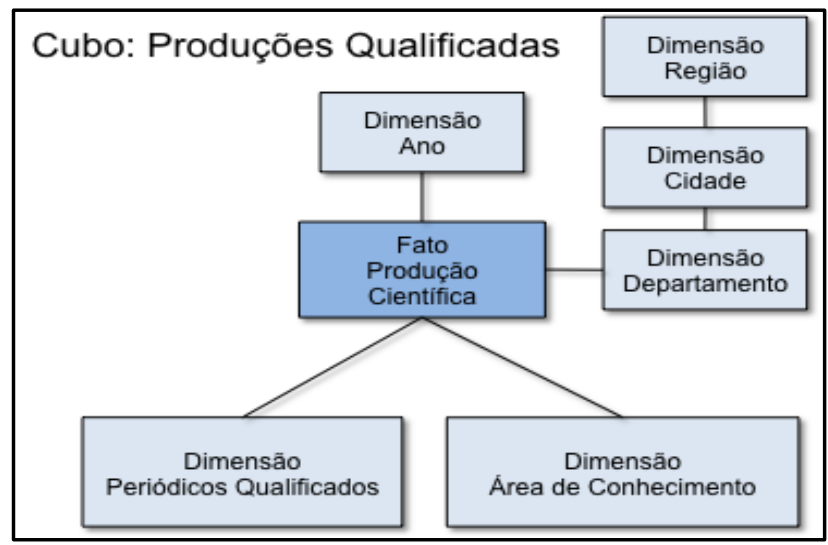

Fonte: Elaborado pelos autores. 
O terceiro cubo Produtividade Docente, figura 4, contempla a estratificação das atividades de pesquisa dos docentes da UNEB nos critérios quantidade de orientações de alunos na iniciação científica, participação em programas de pós-graduação, quantidade de projetos de pesquisa, quantidade de produções de artigos qualificados e quantidade de propriedades intelectuais. Neste caso também foi feito um recorte temporal dos últimos 5 (cinco) anos. Todos estes critérios são atributos quantificadores do Fato Produções Docente.

Figura 4 - Esquema com os elementos do cubo Produtividade Docente.

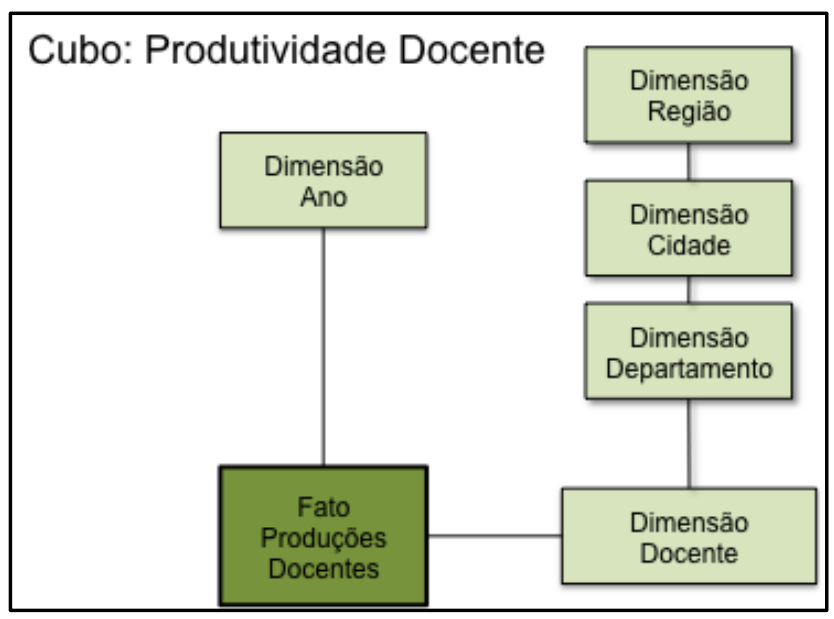

Fonte: Elaborada pelos autores.

\subsection{Aplicação do Modelo}

Para a aplicação do modelo, definido na seção anterior, foi implementado um processo de ETL obtendo dados das fontes enumeradas na etapa II da metodologia (ver seção 4). Com os três cubos povoados de dados, a próxima etapa foi conectar ao banco de dados a ferramenta OLAP Knowage. Através da ferramenta foi possível uma visualização interativa dos dados apresentados na forma de metáforas visuais em gráficos de diversos modelos e tabelas dispostos em painéis temáticos seguindo o domínio dos 3 (três) cubos modelados.

A visualização interativa foi alcançada por meio de filtros e drills que quando aplicados permitem mudar a perspectiva da apresentação dos dados. No caso dos filtros existe um que é predominante em todos os painéis 
que restringe a visualização dos dados no ano corrente. Os drills atuam como uma restrição guiada por meio da hierarquia Região->Cidade$>$ Departamento, onde as dimensões podem ser alternadas conforme a necessidade ou nível de detalhamento exigido para uma determinada situação a ser analisada.

Para ilustrar a dinâmica das consultas realizadas pelos gestores da UNEB são apresentadas de forma estática algumas telas dos painéis temáticos com dados do ano de 2019. O painel de bordo do Corpo docente expõe principalmente dados relacionados ao regime e titulação dos docentes através de gráficos em diversas perspectivas.

A figura 5 apresenta uma visão global pela quantidade de docentes por titulação e os dados que podem ser usados como filtro, são eles: região e título dos docentes. A titulação é uma característica que contribui para conhecer a formação acadêmica do corpo docente da UNEB, tendo influência para progredir nas áreas de pesquisa e inovação. Neste painel também existe uma tabela que apresenta dados relacionados aos docentes bolsistas de Produtividade em Pesquisa (PQ) e em Desenvolvimento Tecnológico e Extensão Inovadora (DT).

Figura 5 - Nível 1 do painel de bordo do Corpo Docente.

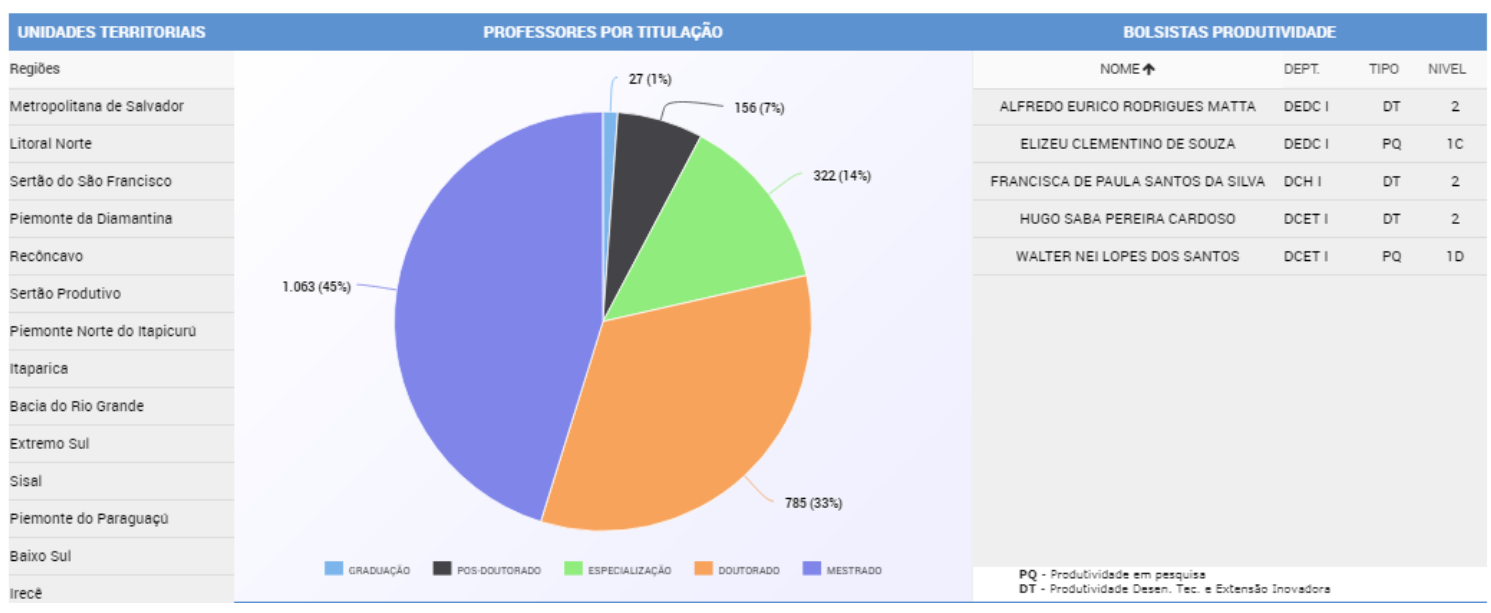

Fonte: Elaborada pelos autores. 
A figura 6 permite visualizar a distribuição dos professores por regime Dedicação Exclusiva (D.E.) em cada departamento do campus da UNEB. O regime de D.E. indica, especificamente, um possível maior envolvimento dos docentes nas atividades de pesquisa e extensão.

\section{Figura 6 - Nível 2 do painel de bordo do Corpo Docente composto pelo gráfico com a quantidade de docentes regime Dedicação Exclusiva (D.E.) em um departamento.}

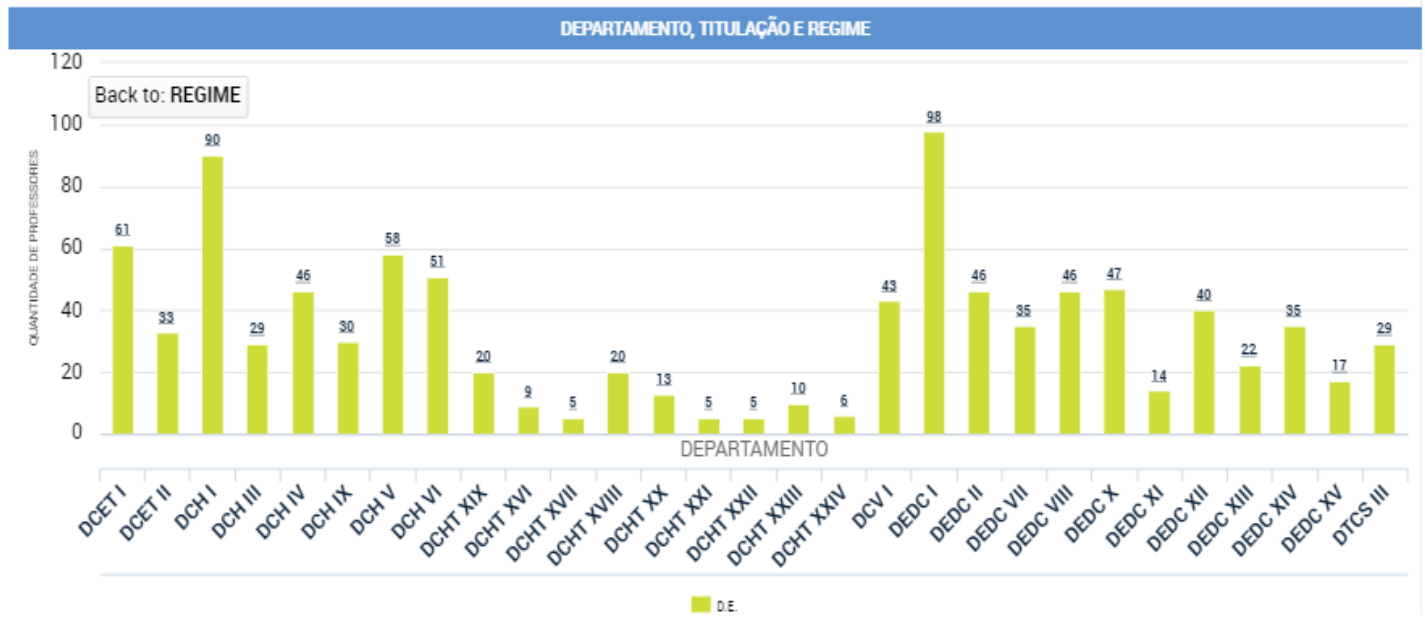

Fonte: Elaborada pelos autores.

O painel de bordo das Produções Qualificadas, apresentado na figura 7, ilustra um gráfico com as produções por qualis de uma área de conhecimento, neste caso o filtro é pela área interdisciplinar, de uma determinada região ou em um nível de detalhe por departamento. Assim, é possível identificar que regiões ou departamentos da UNEB têm um potencial maior de pesquisa ou estruturação de um programa de pós-graduação para uma determinada área de conhecimento.

Outra correlação é estimular áreas de conhecimento que se conectem com a economia local. Por exemplo, é possível identificar o potencial da área de saúde na região metropolitana da UNEB, visto que o departamento de Ciências da Vida fica na cidade Salvador e possui um maior número de pesquisadores, concentrando assim um maior número de publicações $A 1, A 2$ e B1 neste local. 
Figura 7 - Nível 1 do painel de bordo Produções Qualificadas com os gráficos do quantitativo de artigos da área interdisciplinar por região e departamento.

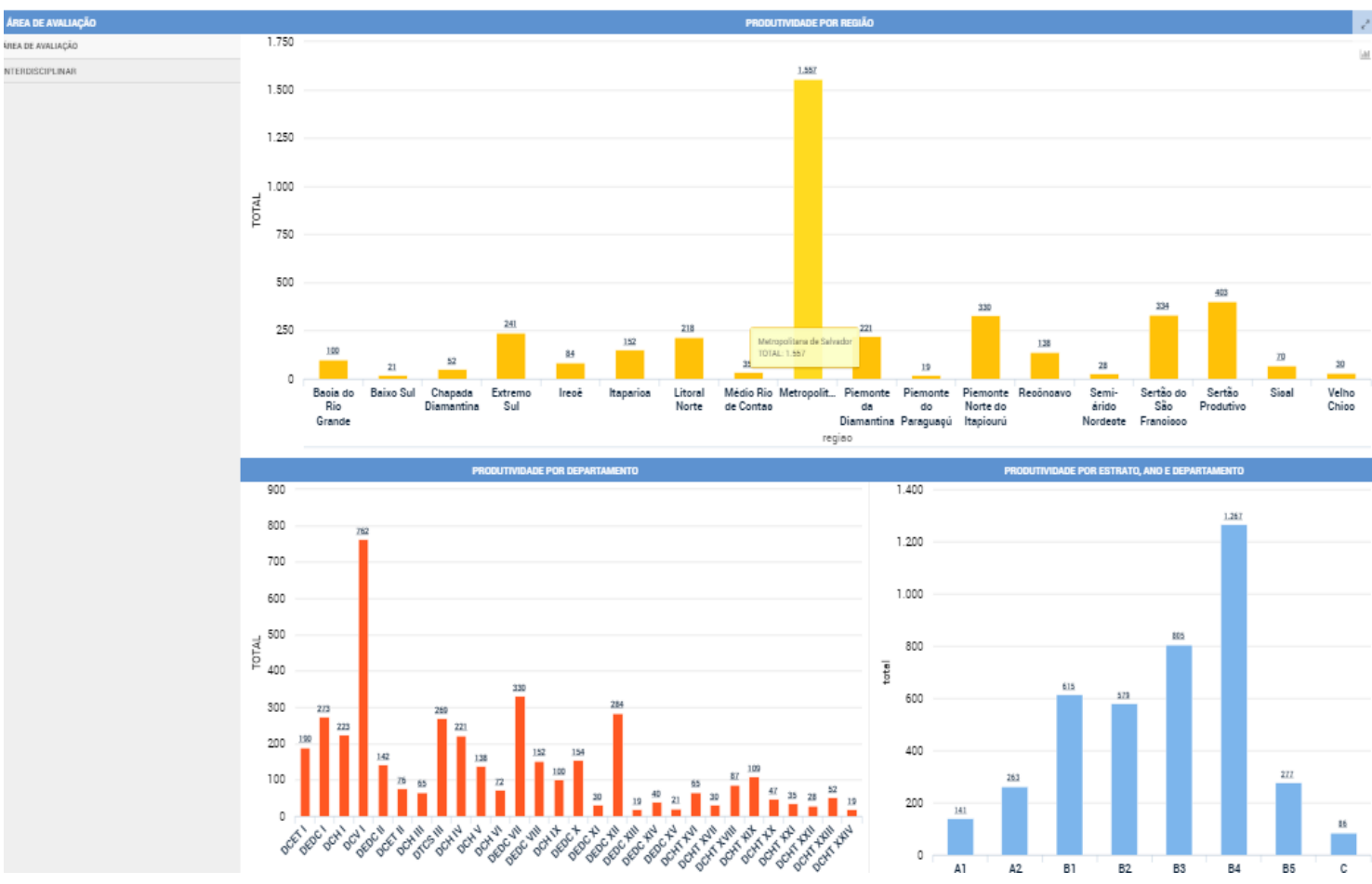

Fonte: Elaborada pelos autores.

O painel Produtividade Docente permite analisar, por exemplo, que regiões ou departamentos possuem uma inatividade menor em relação a pesquisa com docentes que nos últimos 5 (cinco) anos não atuaram nos critérios supracitados no referido Cubo. A figura 8 apresenta um gráfico com 0 quantitativo de cada departamento em relação aos atributos quantificadores filtrado pela região metropolitana. O gráfico apresenta os seguintes atributos quantificadores GP (participação em Grupos de Pesquisa), PP (Projetos de Pesquisa) PG (Participação em Programas de Pós-Graduação), IC (Quantidade de Orientações de Alunos na Iniciação Científica), PER (Quantidade de Artigos em Periódicos) e IN (Quantidade de Propriedades Intelectuais).

Figura 8 - Nível 1 do painel de bordo Produtividade Docente composto pelo gráfico com a totalização relativa aos atributos quantificadores da produtividade dos docentes dos departamentos da região metropolitana. 


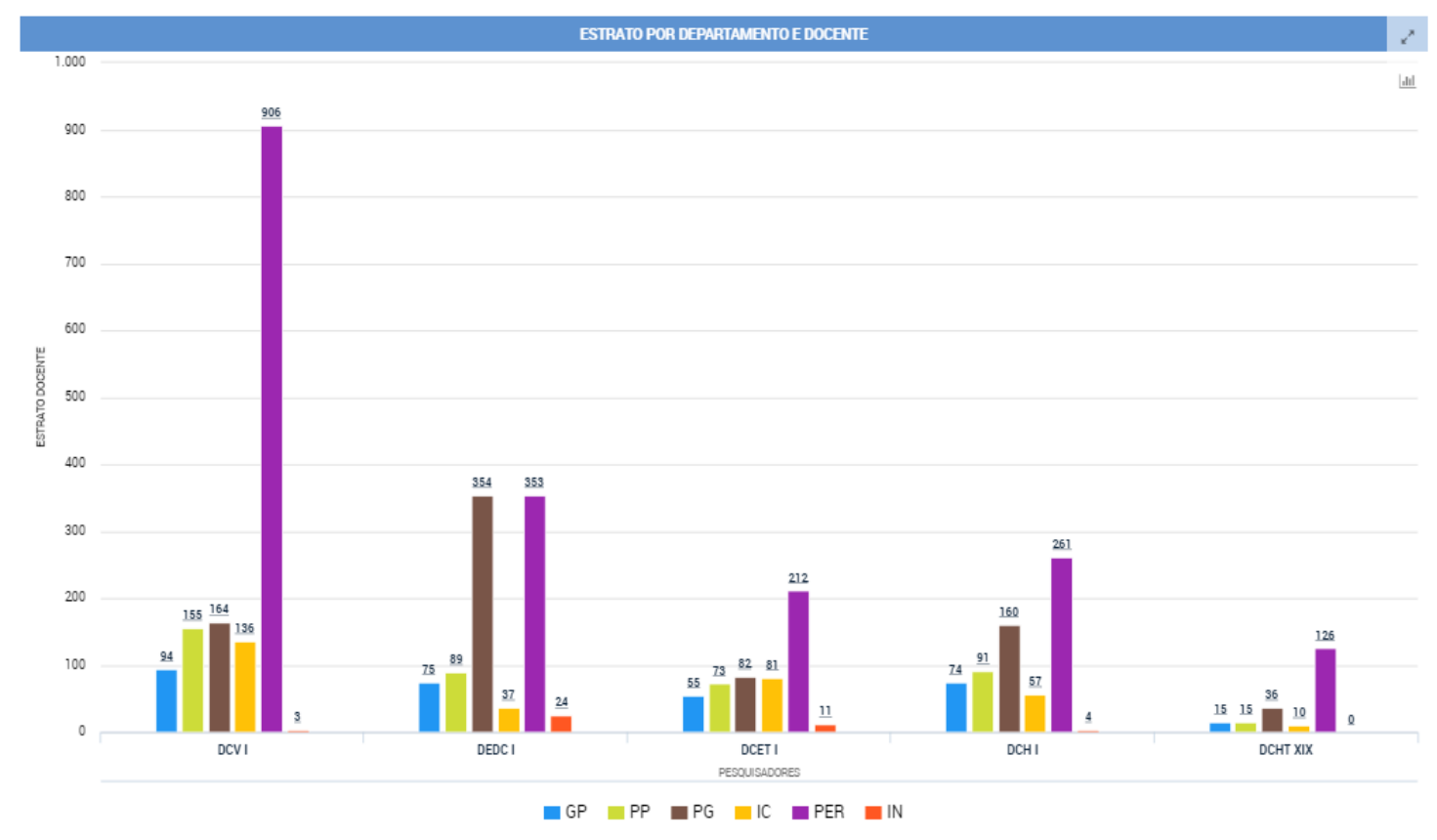

Fonte: Elaborada pelos autores.

\subsection{AnÁlise de Resultados e Discussões}

Esta seção apresenta a última etapa da metodologia onde foram apresentados os painéis temáticos para os gestores da UNEB. Este processo de feedback com os gestores foi aplicado para identificar questões não observadas nas etapas anteriores. $O$ feedback consistiu de entrevistas com gestores onde os mesmos fizeram uso do SAD proposto.

Um ponto em comum para todos foi a importância do SAD para evolução dos indicadores de pesquisa e pós-graduação da UNEB. Os gestores ligados a pós-graduação, através do modelo proposto, fizeram uso do SAD com o foco em analisar quais regiões da Bahia são mais ativas em atividades de pesquisa ou quais departamentos são mais produtivos em determinadas áreas do conhecimento. Outra questão analisada foi se as regiões ou departamentos que possuem mais professores em regime D.E. têm uma maior atividade de pesquisa e pós-graduação. Na apresentação do painel de bordo do Corpo Docente os gestores ligados a pesquisa se interessaram por relações de como os docentes bolsistas de produtividade estão dispostos por titulação, região e ainda, por tipo e categoria da bolsa. Estas relações permitem verificar a qualidade profissional 
dos docentes pesquisadores que influencia diretamente no desempenho da produção científica, e identificar o perfil científico dos docentes e por consequência, o perfil científico de cada região e da UNEB como um todo.

Com a apresentação funcional da ferramenta e a sua dinâmica de interação novos aspectos foram observados em um processo de retroalimentação para evolução do modelo. Os gestores fizeram a sugestão de um conjunto de índices a serem calculados, são eles:

- Total de todas as publicações de um departamento dividido pelo total de professores do departamento;

- Total de todas as publicações de um departamento dividido pelo total de professores do departamento em regime D.E.;

- Total de todas as publicações de um departamento dividido pelo total de professores com titulação de Doutores e Pós-Doutores do departamento;

- Total de professores de um departamento que produziram menos de (10) dez artigos qualificados nos últimos 5 anos;

- Percentual de professores por região ou departamento onde a soma de todos os quantificadores da produtividade docente é igual a zero.

Para atender as sugestões do processo de feedback inicialmente foi analisado se a arquitetura original do SAD com suas dimensões e fatos contemplavam os dados necessários para o cálculo e plotagem dos índices sugeridos. Após esta análise foi observado que o processo de ETL e a camada da base de dados modelada em 3 (três) cubos não tinham necessidade de alteração. Para atender a estes novos requisitos os painéis temáticos foram alterados com novas consultas e gráficos.

\section{CONCLUSÃO E TRABALHOS FUTUROS}

Este artigo apresentou o contexto, os aspectos metodológicos e a arquitetura da informação materializada em solução analítica para integração e visualização de dados relevantes para o mapeamento do cenário da Pesquisa e 
Pós-Graduação da UNEB. Para a concepção da solução analítica foram relevantes as etapas de levantamento de requisitos através da aplicação da estratégia da matriz de necessidade e fontes de dados, definição de indicadores, modelagem de dimensões e suas hierarquias, cubos de dados e 0 desenvolvimento de painéis de bordo.

A materialização de uma solução de gestão analítica se mostrou relevante nas análises dos gestores que buscam potencializar os indicadores de pesquisa da UNEB. Um dessas análises é em relação ao índice RUF de 2017, a UNEB está classificada na $99^{\circ}$ posição, figura em uma posição intermediária considerando os critérios adotados pelo RUF. A compreensão dos indicadores de avaliação das universidades, bem como os dados das dimensões de pesquisa, ensino e extensão, no entanto, podem ser monitorados para o planejamento de ações estratégicas de melhoria.

A gestão destes dados de forma unificada é uma das ações que podem subsidiar os gestores da UNEB a compreenderem as questões prioritárias acerca das áreas de avaliação institucional (indicadores que mensuram as universidades), auxiliando-os na preparação de estratégias que contribuam para o desenvolvimento da universidade. Porém, a realidade atual de distribuição das informações da UNEB ou inexistência de um SAD limitavam os seus gestores. Assim, com as informações integradas no SAD desenvolvido, os gestores da UNEB podem agora fazer campanhas mais precisas de estímulo à pesquisa em regiões ou departamentos que têm priorizado outras ações acadêmicas, como por exemplo, ações extensionistas.

Uma estratégia para validação e evolução do modelo foi a última etapa de feedback. O retorno obtido através da experiência e conhecimento dos gestores da UNEB, somado com uso de uma ferramenta funcional viabilizou a evolução da solução analítica de forma a torná-la ainda mais eficiente com indicadores que balizam os dados em proporções como visto no final da seção anterior.

Como evolução desta pesquisa serão incorporados ao modelo indicadores da inovação e critérios associados à internacionalização que são aspectos importantes para os programas de pós-graduação evoluírem para 
novos patamares. Outras informações relevantes são referentes a startups e as empresas juniores, indicador de grande valor para a inovação e empreendedorismo que como apresentado na introdução é um aspecto de suma importância para realização de pesquisas aplicadas com impacto no desenvolvimento econômico e social.

\section{REFERÊNCIAS}

BARBOSA, A. M. O aperfeiçoamento e desenvolvimento de uma ferramenta tecnológica para disseminação de informações acadêmicas destinadas aos gestores de cursos de graduação da Universidade do Estado da Bahia. 2018. 85 f. Dissertação (Mestrado em Gestão e Tecnologias Aplicadas à Educação - GESTEC) - Departamento de Educação, Universidade do Estado da Bahia (UNEB), Salvador, 2018.

CAPES. Proposta de aprimoramento do modelo de avaliação da PG: Documento final da Comissão Nacional de Acompanhamento do PNPG 2011 2020. 2018. Disponível em:

https://www.capes.gov.br/images/stories/download/conselhosuperior/18102018_PNPG_CS_Avaliacao_Final_CS_FINAL_17_55.pdf. Acesso em: 21 de nov. 2018.

CAPES. Relatório de avaliação: Ensino. 2017. Disponível em: https://capes.gov.br/images/stories/download/avaliacao/relatorios-finaisquadrienal-2017/20122017-ENSINO-quadrienal.pdf. Acesso em: 21 de nov. 2018.

CIRANI, C. B. S.; CAMPANARIO, M. de A.; SILVA, H. H. M. da. A evolução do ensino da pós-graduação senso estrito no Brasil: análise exploratória e proposições para pesquisa. Avaliação (Campinas), Sorocaba, v. 20, n. 1, p. 163-187, mar. 2015.

CWUR. Center for World University Rankings 2018-2019. Disponível em: https://cwur.org/2018-19.php. Acesso em: 18 de nov. 2018.

EXPERTA, Stela. Stela Experta@. Disponível em: http://site.stelaexperta.com.br/. Acesso em: 17 de dez. 2018.

GIL, A. C. Como elaborar projeto de pesquisa. 6 ed. São Paulo: Atlas, 2017. $192 \mathrm{p}$.

KNOWAGE. The Open Source Suite for any modern Business Analytics. Disponível em: https://www.knowage-suite.com/site/home/. Acesso em: 24 de nov. 2018. 
MAGALHÃES, J. L.; QUONIAM, L.; MENA-CHALCO, J. P.; SANTOS, A. Extração e tratamento de dados na base Lattes para identificação de Core Competencies em Dengue. Informação \& Informação, Londrina, v. 19, n. 3, p. 30 - 54, ago. 2014.

MARIADB. About MariaDB. Disponível em: https://mariadb.org/about/. Acesso em: 24 de nov. 2018.

OLIVEIRA, G. L. de. GESMOODLE - Ferramenta de mediação tecnológica e acompanhamento do processo de aprendizagem dos alunos em cursos de graduação a distância da Universidade do Estado da Bahia (UNEB). 2013. 117 f. Dissertação (Mestrado em Gestão e Tecnologias Aplicadas à Educação GESTEC) - Departamento de Educação, Universidade do Estado da Bahia (UNEB), Salvador, 2013.

RUF. Ranking Universitário Folha 2017. Disponível em: http://ruf.folha.uol.com.br/2017/ranking-de-universidades/. Acesso em: 18 de nov. 2018.

VALLEJERA, D.; LOZANO-DÍAZ, I.; RODRIGUEZ-SÁNCHEZ, Y. Bibliometric analysis in a cuban university as a tool for business intelligence. Perspectivas em Gestão \& Conhecimento, João Pessoa, v. 6, n. especial, p. 217-229, jan. 2016.

VERCELLIS, C. Business Intelligence: Data Mining and Optimization for Decision Making. 1 ed. Southern Gate, Chichester, West Sussex: John Willey \& Sons, Ltda, 2009. 420 p.

\title{
ANALYTICAL INFORMATION ARCHITECTURE FOR THE INTEGRATION OF RESEARCH AND GRADUATE PROGRAMS DATA: A CASE STUDY FROM BAHIA STATE UNIVERSITY
}

\begin{abstract}
Introduction: Universities need to monitor their indicators to elaborate stimulus strategies for research and graduate programs. The Bahia State University (UNEB) has similar environment to other universities where there is a dispersion of the data due to the lack of the integration of internal and external systems such as the Lattes Platform and Sucupira Platform systems of the National Council for Scientific and Technological Development (CNPq) and the Coordination for the Improvement of Higher Education Personnel (CAPES) respectively. The data redundancy, inconsistent and unconsolidated information make it difficult for managers to correlate data for analysis of evaluation indicators. Objective: A proposal of information architecture materialized in analytical solution for integration and visualization of data relevant to the mapping of the scenario of the Research and Graduate Programs of the UNEB. Methodology: For this project the methodology is based on the experimental research and the methodology of case
\end{abstract}


study. Strategies such as the need matrix for the lifting of the information layer and the feedback stage through interviews with the UNEB managers have been added to the stages of preparation of the provisional plan of the subject, as well the search for data sources and creation and development of the System of Decision Support (SAD). Results: A data model with three thematic cubes materialized in panels with visual metaphors in tables and interactive graphics allowing filters and drill queries was obtained. Conclusions: The materialization of an analytical management solution proved to be relevant in the analyzes of the managers who seek to enhance the research indicators of the UNEB. With developed SAD, UNEB managers can now conduct more precise campaigns to stimulate research in regions or departments.

Descriptors: Information Architecture. Decision Support System. Academic Indicators.

\title{
ARQUITECTURA DE LA INFORMACIÓN ANALÍTICA PARA LA INTEGRACIÓN DE DATOS DE LA INVESTIGACIÓN Y POSTGRADO: UN ESTUDIO DE CASO DE LA UNIVERSIDAD DEL ESTADO DE BAHÍA
}

\begin{abstract}
RESUMEN
Introducción: Las universidades necesitan monitorear sus indicadores e informaciones para la elaboración de estrategias de estímulo a la investigación y el postgrado. La Universidad del Estado de Bahía (UNEB) tiene un ambiente similar a otras universidades donde existe una dispersión de los datos debido a la falta de integración de sistemas internos y externos como los sistemas de la Plataforma Lattes y de la Plataforma Sucupira del Consejo Nacional del Desarrollo Científico y Tecnológico (CNPq) y de la Coordinación de Perfeccionamiento de Personal de Nivel Superior (CAPES) respectivamente. La redundancia de datos, informaciones incoherentes y no consolidadas dificultan a los gerentes en el proceso de correlación de datos para realizar el análisis de los principales indicadores de evaluación. Objetivo: Uma proposta de arquitetura da informação materializada em solução analítica para integração e visualização de dados relevantes para o mapeamento do cenário da Pesquisa e PósGraduação da UNEB. Metodología: Para este proyecto la metodología está fundamentada en la investigación experimental y en la metodología de estudio de caso. Estrategias como la matriz de necesidad para el levantamiento de la capa de información y la etapa de retroalimentación a través de entrevistas con los gestores de la UNEB se sumaron a etapas de elaboración del plan provisional del asunto así como la búsqueda por fuentes de datos y creación y desarrollo del Sistema de Apoyo a la Decisión (SAD). Resultados: Se obtuvo un modelo de datos con tres cubos temáticos materializados en paneles con metáforas visuales en tablas y gráficos interactivos permitiendo filtros y consultas en drill. Conclusiones: La materialización de una solución de gestión analítica se mostró relevante en los análisis de los gestores que buscan potenciar los indicadores de investigación de la UNEB. Con el SAD desarrollado, los gestores de la UNEB pueden ahora hacer campañas más precisas de estímulo a la investigación en regiones o departamentos.
\end{abstract}

Descriptores: Arquitectura de la información. Sistema de Apoyo a la Decisión. Indicadores Académicos. 
Eduardo Manuel de Freitas Jorge, Franciele Portugal dos Santos, Breno Pádua Brandão Carneiro, Fernanda Almeida Machado

Arquitetura da Informação Analítica para Integração de Dados da Pesquisa e Pós-Graduação: Um Estudo de Caso da Universidade do Estado da Bahia

Recebido em: 03/06/2019

Aceito em: 23/03/2020 